\title{
MONOTONE AND BOOLEAN UNITARY BROWNIAN MOTIONS
}

\author{
TAREK HAMDI
}

\begin{abstract}
The additive monotone (resp. boolean) unitary Brownian motion is a non-commutative stochastic process with monotone (resp. boolean) independent and stationary increments which are distributed according to the arcsine law (resp. Bernoulli law). We introduce the monotone and boolean unitary Brownian motions and we derive a closed formula for their associated moments. This provides a description of their spectral measures. We prove that, in the monotone case, the multiplicative analog of the arcsine distribution is absolutely continuous with respect to the Haar measure on the unit circle, whereas in the boolean case the multiplicative analog of the Bernoulli distribution is discrete. Finally, we use quantum stochastic calculus to provide a realization of these processes as the stochastic exponential of the correspending additive Brownian motions.
\end{abstract}

\section{INTRODUCTION}

In non-commutative probability theory, there exist several natural notions of independence. The most famous ones are the usual independence and the free independence. Other interesting examples are monotone and boolean independence. These allow to define new convolutions for probability measures. The monotone convolutions on the unit circle and the positive half-line were introduced by Bercovici in [2, see also [6]. But the additive monotone Brownian motion and the monotone Fock space where first studied by Muraki, see [9]. The monotone stochastic calculus can also be realized on the symmetric Fock space, see [5]. Bercovici studied also the boolean convolution for probability measures on the positive half-line, but it is not always defined. For probability measures on the unit circle, the boolean convolution is well defined. It was introduced by Franz in [7], see also [3]. The boolean stochastic calculus has been studied by Ben Ghorbal and Schürmann, see [1].

The aim of this paper is to point out several connections between classical, free Brownian motions and their counterparts in the monotone and boolean cases. We shall consider two kinds of unitary Brownian motions, the monotone and boolean one. Both are non-commutative unitary processes, that is families of noncommutative random variables which are unitary and are characterized as having

2010 Mathematics Subject Classification. Primary 60J65, 46L51, 46L53; Secondary 65C30.

Key words and phrases. Convolution semi-groups, monotone and boolean independence, unitary Brownian motion, boson Fock space, boolean Fock space, quantum stochastic calculus. 
independent increments, distributed according to a multiplicative convolution semi-group of measures depending on the notion of independence that we use (monotone or boolean). It has been shown that the additive monotone Brownian motion is distributed according to the arcsine law (cf. [9])

$$
\frac{1}{\pi \sqrt{2 t-x^{2}}} \mathbf{1}_{(-\sqrt{2 t}, \sqrt{2 t})} \mathrm{d} x, \quad t>0 .
$$

Whereas the additive Boolean Brownian motion is Bernoulli distributed (cf. [12])

$$
\frac{1}{2}\left(\delta_{-\sqrt{t}}+\delta_{\sqrt{t}}\right), \quad t>0
$$

So the distributions of the monotone and boolean unitary Brownian motions are a kind of multiplicative analog of these two distributions. We shall next consider the Fock space realization of both unitary Brownian motion by solving the exponential stochastic differential equation

$$
d U_{t}=i d X_{t} U_{t}-\frac{1}{2} U_{t} d t, \quad U_{0}=I
$$

where $\left(X_{t}\right)_{t}$ is the Fock space realization of the corresponding additive Brownian motion. Note that this equation is formally the same as the one used to construct the free unitary Brownian motion (cf. [4]).

This paper is organized as follows. In part one, we first introduce the monotone unitary Brownian motion. We then derive a closed formula for its moments through Legendre polynomials and supply a full description of its distribution, say $\mu_{t}$. In particular, $\mu_{t}$ is compactly-supported and absolutely continuous with respect to the Haar measure on the unit circle. Moreover, it's support is inside an interval of the unit circle, centered at 1 , and spreads as $t$ increases. It only becomes the whole circle asymptotically as $t$ goes to infinity. We close the first part by giving the boson Fock space realization of the monotone unitary Brownian motion. The second part is concerned with boolean unitary Brownian motion. We first introduce this process and we derive an explicit formula for its moments which involves Laguerre polynomials. It turns out that the distribution of the boolean unitary Brownian motion, say $\nu_{t}$, is discrete. Its support is a countably set of points on the unit circle which accumulate at 1. Finally, we recall some basic facts from [1] and we give a Fock space realization of the boolean unitary Brownian motion.

\section{Monotone case}

1.1. Multiplicative monotone convolution. Let $\mu_{1}, \mu_{2}$ be probability measures on the unit circle and set

$$
\psi_{\mu_{i}}=\int_{\mathbb{T}} \frac{z x}{1-z x} \mathrm{~d} \mu_{i}(x), \quad K_{\mu_{i}}(z)=\frac{\psi_{\mu_{i}}(z)}{1+\psi_{\mu_{i}}(z)},
$$


then the multiplicative monotone convolution of $\nu=\mu_{1} \triangleright \mu_{2}$ is uniquely determined by

$$
K_{\nu}(z)=K_{\mu_{1}}\left(K_{\mu_{2}}(z)\right) .
$$

The monotone convolution semigroups of probability measures on the unit circle are given by solutions of the differential equation

$$
\frac{\mathrm{d} K_{t}}{\mathrm{~d} t}(z)=-K_{t}(z) u\left(K_{t}(z)\right)
$$

with initial condition $K_{0}(z)=z$, where $u$ is a holomorphic function on the unit disk with non-negative real part. Therefore $u$ has a Herglotz representation

$$
u(z)=i b+\int \frac{x+z}{x-z} \mathrm{~d} \rho(x)
$$

with $b$ a real number and $\rho$ a finite measure supported on the unit circle.

1.2. Monotone unitary Brownian motion. The distribution $\mu_{t}$ of the unitary monotone Brownian motion correspond to the case $b=0$ and $\rho=\frac{1}{2} \delta_{1}$, i.e. the transform $\left(K_{\mu_{t}}\right)_{t \geq 0}$ is given by the solution of the differential equation

$$
2 \frac{\mathrm{d} K_{\mu_{t}}}{\mathrm{~d} t}(z)=-K_{\mu_{t}}(z) \frac{1+K_{\mu_{t}}(z)}{1-K_{\mu_{t}}(z)}
$$

with initial condition $K_{\mu_{0}}(z)=z$. If we write $Z_{t}=K_{\mu_{t}}$, then the differential equation (1.1) becomes

$$
2 \dot{Z}_{t}=\frac{Z_{t}^{2}+Z_{t}}{Z_{t}-1}
$$

or

$$
\left(\frac{2}{Z_{t}+1}-\frac{1}{Z_{t}}\right)=\frac{1}{2}
$$

which can be integrated to

$$
\frac{t}{2}=\int_{z}^{Z_{t}}\left(\frac{2}{u+1}-\frac{1}{u}\right) \mathrm{d} u=2 \ln \frac{Z_{t}+1}{z+1}-\ln \frac{Z_{t}}{z} .
$$

Exponentiating yields

$$
\frac{\left(Z_{t}+1\right)^{2}}{Z_{t}}=e^{t / 2} \frac{(z+1)^{2}}{z} .
$$

We set $w=e^{t / 2} \frac{(z+1)^{2}}{z}$ and solve the quadratic equation

$$
Z_{t}^{2}+(2-w) Z_{t}+1=0
$$

for $Z_{t}$. This gives

$$
Z_{t}=\frac{1}{2}\left(w-2 \pm \sqrt{w^{2}-4 w}\right)
$$


where the sign \pm is such that $Z_{t}$ is inside the unit disk and $\lim _{t \searrow 0}=z$. But since the only bounded solution of (1.2) is what corresponds to a minus sign, then we get

$$
Z_{t}=\frac{1}{2}(w-2-\sqrt{w(w-4)}) .
$$

Now the moment generating function

$$
\psi_{\mu_{t}}(z):=\sum_{k=1}^{\infty} z^{k} \int_{\mathbb{T}} x^{k} \mathrm{~d} \mu_{t}(x)=\int_{\mathbb{T}} \frac{x z}{1-x z} \mathrm{~d} \mu_{t}(x)
$$

of the distribution of unitary monotone Brownian motion can be recovered as

$$
\psi_{\mu_{t}}=\frac{Z_{t}}{1-Z_{t}}
$$

Note in passing that the family $\left(K_{t}\right)_{t \geq 0}$ defined by $K_{t}(z)=\left.Z_{t}\right|_{Z_{0}=z}$ is a family of self maps of the unit disk which fixes the origin, i.e., $K_{t}(0)=0$. Besides it forms a continuous composition semi-group and it is a general feature that these composition semi-group have such a form.

Remark. Note that the equality (1.3) can also be written as

$$
Z_{t}(z)=\varphi^{-1}\left(e^{t / 2} \varphi(z)\right)
$$

where $\varphi$ is the conformal bijection from $\mathbb{D}$ to $\mathbb{C} \backslash[0,4]$ given by $\varphi(z)=(z+1)^{2} / z$.

\subsection{Moments of $\mu_{t}$.}

Proposition 1. For every $t>0$, one has

$$
\int_{\mathbb{T}} x^{n} \mathrm{~d} \mu_{t}(x)=\frac{1}{2}\left(P_{n}\left(2 e^{-t / 2}-1\right)+P_{n-1}\left(2 e^{-t / 2}-1\right)\right), \quad n \geq 1
$$

where $P_{n}$ is the $n$-th Legendre polynomial.

Proof Since we have

$$
Z_{t}(z)=\frac{1}{2}(w-2-\sqrt{w(w-4)}) \quad z \in \mathbb{D}
$$

with $w=e^{t / 2} \varphi(z)$. Then we obtain

$$
\begin{aligned}
\psi_{\mu_{t}}(z)=\frac{Z_{t}}{1-Z_{t}} & =-\frac{w-\sqrt{w(w-4)}-2}{w-\sqrt{w(w-4)}-4} \\
& =-1+\frac{-2}{w-\sqrt{w(w-4)}-4} .
\end{aligned}
$$

Writing

$$
\frac{1}{w-\sqrt{w(w-4)}-4}=\frac{-1}{4}\left(1+\sqrt{\frac{w}{w-4}}\right),
$$


we get

$$
\psi_{\mu_{t}}(z)=-\frac{1}{2}+\frac{z+1}{2 \sqrt{1-2 z\left(2 e^{-t / 2}-1\right)+z^{2}}} .
$$

Now using the generating function for Legendre polynomial

$$
\frac{1}{\sqrt{1-2 x z+z^{2}}}=\sum_{n \geq 0} P_{n}(x) z^{n}
$$

we obtain the following expansion

$$
\begin{aligned}
\psi_{\mu_{t}}(z) & =-\frac{1}{2}+\frac{z+1}{2} \sum_{n \geq 0} P_{n}\left(2 e^{-t / 2}-1\right) z^{n} \\
& =-\frac{1}{2}+\frac{P_{0}\left(2 e^{-t / 2}-1\right)}{2}+\frac{1}{2} \sum_{n \geq 1}\left(P_{n}\left(2 e^{-t / 2}-1\right)+P_{n-1}\left(2 e^{-t / 2}-1\right)\right) z^{n} \\
& =\frac{1}{2} \sum_{n \geq 1}\left(P_{n}\left(2 e^{-t / 2}-1\right)+P_{n-1}\left(2 e^{-t / 2}-1\right)\right) z^{n} .
\end{aligned}
$$

Remark. The moments of the monotone unitary BM are real. This is due to the fact that the distribution of the unitary monotone $B M$ is symmetric, i.e., $U_{t}$ and $U_{t}^{*}$ have the same distribution.

\subsection{Description of $\mu_{t}$.}

Theorem 1. For any $t>0$, the measure $\mu_{t}$ is absolutely continuous with respect to the Haar measure on $\mathbb{T}$. Its support is equal to the interval

$$
I_{t} \triangleq\left\{e^{i \theta} \mid-\arccos \left(2 e^{-t / 2}-1\right) \leq \theta \leq \arccos \left(2 e^{-t / 2}-1\right)\right\}
$$

where arccos $\in[0, \pi]$. The density is positive on the interior of $I_{t}$ and is equal to

$$
\frac{\sqrt{2} \cos (\theta / 2)}{\sqrt{\cos (\theta)-\left(2 e^{-t / 2}-1\right)}} 1_{\left\{\cos (\theta)>2 e^{-t / 2}-1\right\}}, \quad \theta \in(-\pi, \pi) .
$$

Proof Let $H_{\mu_{t}}$ be the Herglotz transform of $\mu_{t}$. We have

$$
H_{\mu_{t}}(z)=1+2 \psi_{\mu_{t}}(z)=\frac{z+1}{\sqrt{1-2 z\left(2 e^{-t / 2}-1\right)+z^{2}}}, \quad|z|<1 .
$$

Since $z \mapsto 1-2 z\left(2 e^{-t / 2}-1\right)+z^{2}$ does not take negative value and its roots $a_{t}, \overline{a_{t}}$ lie on $\mathbb{T}$ with $a_{t}=e^{i \arccos \left(2 e^{-t / 2}-1\right)}$. Then $H_{\mu_{t}}$ is analytic in the open disc and it extends continuously to $\mathbb{T} \backslash\left\{a_{t}, \overline{a_{t}}\right\}$. Furthermore for $\theta_{t}=\arccos \left(2 e^{-t / 2}-1\right)$, we have

$$
\lim _{r \rightarrow 1, z=r e^{ \pm i \theta_{t}}}\left(z-e^{ \pm i \theta_{t}}\right) H_{\mu_{t}}(z)=0
$$


In fact, for any $z=r e^{ \pm i \theta_{t}}$, we write

$$
\begin{aligned}
\left(z-e^{ \pm i \theta_{t}}\right) H_{\mu_{t}}(z) & =\left(z-e^{ \pm i \theta_{t}}\right) \frac{z+1}{\sqrt{\left(z-a_{1}\right)\left(z-a_{2}\right)}} \\
& =\left(z-e^{ \pm i \theta_{t}}\right) \frac{z+1}{\sqrt{\left(z-e^{i \theta_{t}}\right)\left(z-e^{-i \theta_{t}}\right)}} \\
& =\frac{(r-1) e^{ \pm i \theta_{t}}\left(e^{ \pm i \theta_{t}}+1\right)}{\sqrt{r-1} \sqrt{r e^{ \pm 2 i \theta_{t}}-1}} \\
& =\frac{\sqrt{r-1} e^{ \pm i \theta_{t}}\left(e^{ \pm i \theta_{t}}+1\right)}{\sqrt{r e^{ \pm 2 i \theta_{t}}-1}}
\end{aligned}
$$

Then $\mu_{t}$ is absolutely continuous with respect to the Haar measure on the unit circle, with density given by the Poisson kernel

$$
P_{\mu_{t}}\left(e^{i \theta}\right)=\Re\left[H_{\mu_{t}}\left(e^{i \theta}\right)\right], \quad \theta \in(-\pi, \pi) .
$$

Now we need to compute $\Re\left[H_{\mu_{t}}(w)\right]$ for $w \in \mathbb{T}$. We have

$$
\begin{aligned}
H_{\mu_{t}}(w) & =\frac{w+1}{\sqrt{1-2 w\left(2 e^{-t / 2}-1\right)+w^{2}}} \\
& =\frac{w+1}{\sqrt{w} \sqrt{w+\bar{w}-2\left(2 e^{-t / 2}-1\right)}} 1_{\left\{(w+\bar{w})-2\left(2 e^{-t / 2}-1\right)>0\right\}} \\
& =\frac{(w+1)(\sqrt{(2+w+\bar{w}) / 4}-i \sqrt{(2-w-\bar{w}) / 4})}{\sqrt{w+\bar{w}-2\left(2 e^{-t / 2}-1\right)}} 1_{\left\{(w+\bar{w})-2\left(2 e^{-t / 2}-1\right)>0\right\}} \\
& =\frac{(2+w+\bar{w}+(w-\bar{w}))(\sqrt{(2+w+\bar{w}) / 4}-i \sqrt{(2-w-\bar{w}) / 4})}{2 \sqrt{w+\bar{w}-2\left(2 e^{-t / 2}-1\right)}} 1_{\left\{(w+\bar{w})-2\left(2 e^{-t / 2}-1\right)>0\right\}} .
\end{aligned}
$$

Hence we get

$$
P_{\mu_{t}}\left(e^{i \theta}\right)=\frac{\sqrt{2} \cos (\theta / 2)}{\sqrt{\cos (\theta)-\left(2 e^{-t / 2}-1\right)}} 1_{\left\{\cos (\theta)>2 e^{-t / 2}-1\right\}}
$$

Remark. Note that for classical Brownian motion the support is immediately the whole circle and in the free case, it is an interval smaller then the circle for $t<4$ and the whole circle for $t \geq 4$. Whereas, for the monotone case it remains smaller than the circle for all $t$, it only becomes the whole circle asymptotically as $t$ goes to infinity.

\subsection{Quantum stochastic calculus and monotone unitary Brownian mo- tion.}


1.5.1. Quantum stochastic calculus. We shall recall some basic facts from quantum stochastic calculus in a boson Fock space as expounded in [10]. Let $H:=\Gamma\left(L^{2}\left(\mathbb{R}_{+}\right)\right)$be the boson Fock space with one degree of freedom and let $A^{*}, A$ be respectively the creation and annihilation operators on $H$. For each $t \in \mathbb{R}_{+}$we have the following identification

$$
\Gamma\left(L^{2}\left(\mathbb{R}_{+}\right)\right) \cong \Gamma\left(L^{2}([0, t])\right) \otimes \Gamma\left(L^{2}([t, \infty))\right) .
$$

Let $\Omega_{t]}$ be the Fock vacuum vector in $H_{t]}:=\Gamma\left(L^{2}([0, t])\right)$ and let $P_{0}(t)$ be the projection on the subspace $\Omega_{t]} \otimes H_{[t}$ where $H_{[t}:=\Gamma\left(L^{2}([t, \infty))\right)$. According to [10, Theorem 2.1], the quantum stochastic integrals

$$
L_{t}=\int_{0}^{t} P_{0}(s) d A_{s}, \quad L_{t}^{*}=\int_{0}^{t} P_{0}(s) d A_{s}^{*}
$$

are defined on the whole space $H$ as bounded operators, adjoint to each other and satisfy

$$
L_{t} L_{t}^{*}=\int_{0}^{t} P_{0}(s) d s
$$

Now, let us denote $B_{t}=A_{t}+A_{t}^{*}$ and $X_{t}=L_{t}+L_{t}^{*}$. We recall that the family of operators $\left(X_{t}\right)_{t \in \mathbb{R}_{+}}$on $\Gamma(H)$ is the non-commutative arcsine Brownian motion for the vacuum expectation $\phi()=.\langle e(0), e(0)\rangle$ on $B(\Gamma(H))$, (See [10]).

\subsubsection{Boson Fock space realization of monotone unitary Brownian mo-}

tion. We shall now use quantum stochastic calculus on a boson Fock space in order to give a construction of monotone unitary Brownian motion as the "stochastic exponential" of the monotone additive one. For this we shall solve a stochastic differential equation. According to [8] there exists a unique family of unitary operators $\left(U_{t}\right)_{t \in \mathbb{R}_{+}}$, solution to the stochastic differential equation

$$
d U_{t}=\left(i d X_{t}-\frac{1}{2} P_{0}(t) d t\right) U_{t}, \quad U_{0}=1
$$

¿From this equation we deduce,

Lemma 1. For all $n \geq 1$ and $t \geq 0$ one has

$$
d U_{t}^{n}=i \sum_{l=1}^{n} U_{t}^{n-l} P_{0} U_{t}^{l} d B_{t}-\frac{1}{2} \sum_{l=1}^{n} U_{t}^{n-l} P_{0} U_{t}^{l} d t-\sum_{k=2}^{n} \sum_{l=1}^{k-1} U_{t}^{n-k} P_{0} U_{t}^{k-l} P_{0} U_{t}^{l} d t
$$


Proof We prove the result by induction on $n$. For $n=1$ this reduce to the equation for $U$. Assume the result holds for $n$, then by quantum Ito's formula

$$
\begin{aligned}
d U_{t}^{n+1}= & U_{t} d U_{t}^{n}+d U_{t} U_{t}^{n}+d U_{t} d U_{t}^{n} \\
= & i \sum_{l=1}^{n} U_{t}^{n-l+1} P_{0} U_{t}^{l} d B_{t}-\frac{1}{2} \sum_{l=1}^{n} U_{t}^{n-l+1} P_{0} U_{t}^{l} d t-\sum_{k=2}^{n} \sum_{l=1}^{k-1} U_{t}^{n-k+1} P_{0} U_{t}^{k-l} P_{0} U_{t}^{l} d t \\
& +\left(i P_{0} d B_{t}-\frac{1}{2} P_{0} d t\right) U_{t}^{n+1}-\sum_{l=1}^{n} P_{0} U_{t}^{n-k+1} P_{0} U_{t}^{l} d t \\
= & i \sum_{l=1}^{n+1} U_{t}^{n+1-l} P_{0} U_{t}^{l} d B_{t}-\frac{1}{2} \sum_{l=1}^{n+1} U_{t}^{n+1-l} P_{0} U_{t}^{l} d t-\sum_{k=2}^{n+1} \sum_{l=1}^{k-1} U_{t}^{n+1-k} P_{0} U_{t}^{k-l} P_{0} U_{t}^{l} d t
\end{aligned}
$$

We shall now prove that

Theorem 2. The process $\left(U_{t}\right)_{t \in \mathbb{R}_{+}}$is a monotone unitary Brownian motion.

Proof Form (1.4), we have

$$
U_{t}-U_{s}=i \int_{s}^{t} d X_{r} U_{r}-\frac{1}{2} \int_{s}^{t} P_{0}(r) U_{r} d r
$$

so that,

$$
U_{t} U_{s}^{\star}=1+i \int_{s}^{t} P_{0}(r) U_{r} U_{s}^{\star} d B_{r}-\frac{1}{2} \int_{s}^{t} P_{0}(r) U_{r} U_{s}^{\star} d r
$$

But the kernels of the projections $P_{0}(s)$ increase with $s$, this says that $P_{0}(r)=$ $P_{0}(r) P_{0}(s)$ for any $s \leq r$. Consequently from (1.5), we get

$$
U_{t} U_{s}^{\star}-1=P_{0}(s)\left(U_{t} U_{s}^{\star}-1\right)
$$

and so for all $0 \leq s_{1}<t_{1}<\ldots<t_{n-1}<s_{n}<t_{n}<\infty$ and $k_{1}, \ldots, k_{n} \in \mathbb{N}$, we get

$$
\begin{aligned}
\phi\left(\left(U_{t_{1}} U_{s_{1}}^{\star}-1\right)^{k_{1}} \ldots\left(U_{t_{n}} U_{s_{n}}^{\star}-1\right)^{k_{n}}\right) & =\phi\left(P_{0}\left(s_{1}\right)\left(U_{t_{1}} U_{s_{1}}^{\star}-1\right)^{k_{1}} \ldots P_{0}\left(s_{n}\right)\left(U_{t_{n}} U_{s_{n}}^{\star}-1\right)^{k_{n}}\right) \\
& =\prod_{i=1}^{n} \phi\left(\left(U_{t_{i}} U_{s_{i}}^{\star}-1\right)^{k_{i}}\right) .
\end{aligned}
$$

Which proves that the increments of the process $\left(U_{t}\right)_{t \in \mathbb{R}_{+}}$are monotonically independent with respect to the state $\phi$. Now the equality (1.5) implies that the process $t \mapsto U_{t} U_{s}^{\star}$ is obtained by solving the same equation as $U$ but with respect to the monotone Brownian motion $\left(X_{t}-X_{s}\right)_{t \geq s}$. This says that the increments of $\left(U_{t}\right)_{t \in \mathbb{R}_{+}}$have a stationary distribution. So it is enough to prove that the distribution of $U_{t}$ is $\mu_{t}$ for all $t \geq 0$. To this end, we take the state $\phi$ in both sides of the equation in Lemma 1. Then we get

$$
\frac{d}{d t} \phi\left(U_{t}^{n}\right)=-\frac{1}{2} \sum_{l=1}^{n} \phi\left(U_{t}^{n-l}\right) \phi\left(U_{t}^{l}\right)-\sum_{k=2}^{n} \sum_{l=1}^{k-1} \phi\left(U_{t}^{n-k}\right) \phi\left(U_{t}^{k-l}\right) \phi\left(U_{t}^{l}\right) .
$$


Now, introducing the generating function

$$
\rho(t, z)=\sum_{n=1}^{\infty} \phi\left(U_{t}^{n}\right) z^{n}, \quad|z|<1
$$

we get the differential equation

$$
\partial_{t} \rho(t, z)=-\frac{1}{2} \rho(t, z)(1+\rho(t, z))-\rho(t, z)^{2}(1+\rho(t, z)),
$$

or equivalently

$$
\partial_{t} \rho(t, z)=-\frac{1}{2} \rho(t, z)(1+\rho(t, z))(1+2 \rho(t, z))
$$

After integrating and taking into account $\rho(0, z)=z /(1-z)$, we get

$$
\frac{\rho(t, z)(1+\rho(t, z))}{(1+2 \rho(t, z))^{2}}=\frac{z e^{-\frac{t}{2}}}{(1+z)^{2}}=\frac{1}{\varphi\left(Z_{t}(z)\right)} .
$$

Hence $\rho(t,)=.\psi_{\mu_{t}}$ for any $t \geq 0$ and the distribution of $U_{t}$ is $\mu_{t}$. This ends the proof of Theorem 2 .

\section{Boolean case}

2.1. Multiplicative boolean convolution. Let $\mu_{1}, \mu_{2}$ be probability measures on the unit circle and set

$$
\psi_{\mu_{i}}=\int_{\mathbb{T}} \frac{z x}{1-z x} \mathrm{~d} \mu_{i}(x), \quad F_{\mu_{i}}(z)=\frac{1}{z} \frac{\psi_{\mu_{i}}(z)}{1+\psi_{\mu_{i}}(z)},
$$

then the multiplicative boolean convolution $\nu=\mu_{1} \otimes \mu_{2}$ is uniquely determined by

$$
F_{\nu}(z)=F_{\mu_{1}}(z) F_{\mu_{2}}(z)
$$

For infinitely divisible measures the transform is of the form

$$
F(z)=\exp (u(z))
$$

where $u$ is a holomorphic function on the unit disk with non-positive real part and therefore has a Herglotz representation of the form

$$
u(z)=i b-\int \frac{x+z}{x-z} \mathrm{~d} \rho(x)
$$

with $b$ a real number and $\rho$ a finite measure supported on the unit circle, cf. [7, Remark 3.7]. 
2.2. Boolean unitary Brownian motion. The distribution $\nu_{t}$ of the boolean Brownian motion on the unit circle corresponds again to the case $b=0, \rho=\frac{1}{2} \delta_{1}$, i.e. to the transforms

$$
F_{t}(z)=\exp \left(\frac{t(z+1)}{2(z-1)}\right), \quad t \geq 0
$$

In this case, one gets

$$
\psi_{\nu_{t}}(z)=\frac{z \exp \left(\frac{t(z+1)}{2(z-1)}\right)}{1-z \exp \left(\frac{t(z+1)}{2(z-1)}\right)}
$$

and in a small neighborhood of the origin

$$
\psi_{\nu_{t}}(z)=\sum_{k \geq 1} z^{k} \exp \left(k \frac{t(z+1)}{2(z-1)}\right)=\sum_{k \geq 1}\left(z e^{-\frac{t}{2}}\right)^{k} \exp \left(k t \frac{z}{z-1}\right) .
$$

\subsection{Moments of $\nu_{t}$.}

Proposition 2. For any $t>0$ and any $n \geq 1$, one has

$\int_{\mathbb{T}} x^{n} \mathrm{~d} \nu_{t}(x)=\sum_{k=1}^{n} L_{n-k}^{(1)}(k t) e^{-k t / 2}-2 \sum_{k=1}^{n-1} L_{n-k-1}^{(1)}(k t) e^{-k t / 2}+\sum_{k=1}^{n-2} L_{n-k-2}^{(1)}(k t) e^{-k t / 2}$

where the sums in the RHS are taken to be empty when $k<1$ and $L_{n}^{(1)}$ is the $n$-th Laguerre polynomial of index 1.

Proof We use the generating function for Laguerre polynomials

$$
\exp \left(k t \frac{z}{z-1}\right)=(1-z)^{\alpha+1} \sum_{n \geq 0} L_{n}^{(\alpha)}(k t) z^{n}
$$

where $\alpha>-1$. We can choose $\alpha=1$ and get after inverting the order of summation

$$
\begin{aligned}
\psi_{\nu_{t}}(z) & =(1-z)^{2} \sum_{k \geq 1} e^{-k t / 2} \sum_{n \geq k} L_{n-k}^{(1)}(k t) z^{n} \\
& =(1-z)^{2} \sum_{n \geq 1}\left(\sum_{k=1}^{n} L_{n-k}^{(1)}(k t) e^{-k t / 2}\right) z^{n} .
\end{aligned}
$$

Identifying the coefficients of $z^{n}$, we get the result.

Remark. The moments are again real, because the law of the unitary boolean $B M$ is also symmetric. 
2.4. Description of $\nu_{t}$. Observe that the Herglotz transform $k_{t}$ of $\nu_{t}$ is given by

$$
k_{t}(z)=\int_{\mathbb{T}} \frac{\xi+z}{\xi-z} d \nu_{t}(\xi)=1+2 \psi_{\nu_{t}}(z)=\frac{1+\theta_{t}(z)}{1-\theta_{t}(z)}, \quad t>0, z \in \mathbb{D}
$$

with

$$
\theta_{t}(z)=z \exp \left(\frac{t(z+1)}{2(z-1)}\right)
$$

For $t>0$, let $\Sigma_{t}$ denote the subset of $\mathbb{T}$ consisting of the solutions to the equation $\theta_{t}(\zeta)=1$. We shall need the following lemma.

Lemma 2. $\Sigma_{t}$ is a discrete set of points which accumulate at 1.

Proof Fix $t>0$ and let $\zeta=x+i y \in \Sigma_{t}$. This says that

$$
\exp \left(-\frac{i t y}{2(1-x)}\right)=x-i y \text {. }
$$

By a simple identification of real and imaginary parts we obtain

$$
\left\{\begin{array}{l}
\frac{t y}{2(1-x)}=\epsilon \arccos (x)+2 k \pi, \epsilon \in\{ \pm 1\} \text { and } k \in \mathbb{Z} \\
\sin \left(\frac{t y}{2(1-x)}\right)=y
\end{array}\right.
$$

which in turn leads to

$$
\left\{\begin{array}{l}
\frac{t}{2} \sqrt{\frac{1+x}{1-x}}-\arccos (x)=2 k \epsilon \pi, \epsilon \in\{ \pm 1\} \text { and } k \in \mathbb{Z} \\
y=\epsilon \sqrt{1-x^{2}}
\end{array} .\right.
$$

\begin{tabular}{|c|c|c|c|}
\hline$x$ & -1 & $x_{0}(t)$ & 1 \\
\hline$\partial_{x} g_{t}(x)$ & & + & \\
\hline$g_{t}(x)$ & $-\pi$ & 0 & $+\infty$ \\
\hline
\end{tabular}

Consider now the function $g_{t}(x)=\frac{t}{2} \sqrt{\frac{1+x}{1-x}}-\arccos (x)$ with $x \in[-1,1)$. Then we have

$$
\partial_{x} g_{t}(x)=\frac{t+2-2 x}{2(1-x) \sqrt{1-x^{2}}} \geq 0
$$

and so

Consequently for any $k \in \mathbb{Z}$, the equation $g_{t}(x)=2 k \epsilon \pi$ has a unique solution $x_{k}(t)$ if $\epsilon k \geq 0$ and no solution otherwise. Hence

$$
\Sigma_{t}=\left\{x_{n}(t)+i \sqrt{1-x_{n}(t)^{2}}, n \in \mathbb{N}\right\} \cup\left\{x_{-n}(t)-i \sqrt{1-x_{-n}(t)^{2}}, n \in \mathbb{N}\right\} .
$$

Since $g_{t}$ is injective and we have

$$
g_{t}\left(x_{-n}(t)\right)=2(-n)(-1) \pi=2 n \pi=g_{t}\left(x_{n}(t)\right),
$$


we obtain $x_{n}(t)=x_{-n}(t)$ then

$$
\Sigma_{t}=\left\{\zeta_{ \pm n}(t)=x_{n}(t) \pm i \sqrt{1-x_{n}(t)^{2}}: g_{t}\left(x_{n}(t)\right)=2 n \pi, n \in \mathbb{N}\right\}
$$

It remains to show that $\left(\zeta_{n}(t)\right)_{n \in \mathbb{Z}}$ accumulate at 1 . Equivalently, we shall show that $\lim _{n \rightarrow+\infty} x_{n}(t)=1$. Notice that the sequence $\left(x_{n}(t)\right)_{n \in \mathbb{N}}$ is bounded. Moreover, for $n \leq m$, we have

$$
g_{t}\left(x_{n}(t)\right)=2 \pi n \leq 2 \pi m=g_{t}\left(x_{m}(t)\right) .
$$

Thus $\left(x_{n}(t)\right)_{n \in \mathbb{N}}$ is increasing and so it converges. We put

$$
x(t)=\lim _{n \rightarrow+\infty} x_{n}(t) .
$$

Then we have

$$
-1<x_{0}(t) \leq x(t) \leq 1
$$

But, the equality $g_{t}\left(x_{n}(t)\right)=2 n \pi$ entails $\lim _{n \rightarrow+\infty} g_{t}\left(x_{n}(t)\right)=+\infty$. Whence, by continuity of $g_{t}$, we deduce that $x(t)=1$.

Now, we proceed to the study of $\nu_{t}$. Observe that for $z \in \mathbb{D}$,

$$
\left|z \exp \left(\frac{t(z+1)}{2(z-1)}\right)\right|=|z| \exp \left(\frac{t}{2} \Re\left(\frac{z+1}{z-1}\right)\right)=|z| \exp \left(-\frac{t\left(1-|z|^{2}\right)}{2|z-1|^{2}}\right)<1,
$$

so $\theta$ is an analytic self-map of $\mathbb{D}$. From the previous identity one can easily check that $\left|\theta_{t}(\xi)\right|=1$ if $\xi \in \mathbb{T}$. Thus $\theta_{t}$ is an inner function and hence $\nu_{t}$ is singular with respect to the Haar measure on the unit circle. Moreover, $\nu_{t}$ coincides with the Aleksandrov-Clark measure associated to $\theta_{t}$ at 1 (cf. [11]). Define the Poisson transform of $\nu_{t}$ by

$$
P_{\nu_{t}}(z):=\Re\left[k_{t}(z)\right]=\int_{\mathbb{T}} \frac{1-r^{2}}{|\xi-z|^{2}} d \nu_{t}(\xi), \quad t>0 .
$$

Let $z=r \zeta$, where $r \in(0,1)$ and $\zeta \in \mathbb{T}$, then the above expression becomes

$$
P_{\nu_{t}}(r \zeta)=\int_{\mathbb{T}} \frac{1-r^{2}}{|\xi-r \zeta|^{2}} d \nu_{t}(\xi)=\frac{1-\left|\theta_{t}(r \zeta)\right|^{2}}{\left|1-\theta_{t}(r \zeta)\right|^{2}}, \quad t>0
$$

The rightmost term approaches zero as $r \rightarrow 1$ except for the set of solutions to the equation $\theta_{t}(\zeta)=1$. By Lemma 2, this set is equal to $\left(\zeta_{n}\right)_{n \in \mathbb{Z}}$. Which says that $\nu_{t}$ is placing no mass on $\mathbb{T} \backslash\left\{\left(\zeta_{n}\right)_{n \in \mathbb{Z}}\right\}$ and so (cf. [11, Proposition 4.14])

$$
\nu_{t}=\sum_{n \in \mathbb{Z}} c_{n}(t) \delta_{\zeta_{n}}, \quad t>0
$$

where $c_{n}(t)$ are positive constants given by

$$
c_{n}(t)=\frac{1}{\left|\theta_{t}^{\prime}\left(\zeta_{n}\right)\right|}=\frac{\left|\zeta_{n}-1\right|^{2}}{\left|\overline{\zeta_{n}}\left(\zeta_{n}-1\right)^{2}-t\right|}
$$


Then by writing $\zeta_{n}=e^{i \alpha_{n}}$, we obtain

So we have

$$
c_{n}(t)=\frac{2\left(1-\cos \alpha_{n}\right)}{t+2\left(1-\cos \alpha_{n}\right)} \text {. }
$$

$$
\nu_{t}=\sum_{n \in \mathbb{Z}} \frac{2\left(1-\cos \alpha_{n}\right)}{t+2\left(1-\cos \alpha_{n}\right)} \delta_{e^{i \alpha_{n}}}, \quad t>0 .
$$

Remark. Since $\left(x_{n}(t)\right)_{n \in \mathbb{N}}$ is increasing, then one can see that

$$
\operatorname{supp} \nu_{t} \subset\left\{e^{i \theta} \mid-\arccos \left(x_{0}(t)\right) \leq \theta \leq \arccos \left(x_{0}(t)\right)\right\}
$$

where $\arccos \in[0, \pi]$.

Next, we shall show that the above interval becomes the whole unit circle asymptotically as $t$ goes to infinity.

Proposition 3. One has

$$
\lim _{t \rightarrow+\infty} x_{0}(t)=-1
$$

Proof Since $\partial_{x} g_{t}$ does not vanishes for any fixed $(t, x) \in \mathbb{R}_{+}^{*} \times[-1,1]$, the implicit function theorem implies that there exists a continuously differentiable function $\varphi$ such that $x_{0}=\varphi(t)$ and

$$
\varphi^{\prime}(s)=-\frac{\partial_{t} g_{t}(s, x)}{\partial_{x} g_{t}(s, x)}=\frac{x^{2}-1}{s+1-x} \leq 0
$$

for any $s$ in an open neighborhood of $t$ in $\mathbb{R}_{+}^{*}$. It follows that $x_{0}$ is decreasing with respect to $t$ and thus the limit of $x_{0}$ as $t \rightarrow+\infty$ exists. Let $l=\lim _{t \rightarrow+\infty} x_{0}(t)$, then $l \in[-1,1)$. But since $g_{t}\left(x_{0}(t)\right)=0$, then we get

$$
\sqrt{\frac{1+x_{0}(t)}{1-x_{0}(t)}}=\frac{2 \arccos \left(x_{0}(t)\right)}{t} .
$$

The LHS of the previous identity goes to zero as $t \rightarrow+\infty$. Thus the RHS must do the same. This gives us $\sqrt{(1+l) /(1-l)}=0$, so we have $l=-1$.

\subsection{Boolean stochastic calculus and boolean unitary Brownian motion.}

2.5.1. Boolean stochastic calculus. We shall recall the relevant facts from boolean stochastic calculus as expounded in [1]. For a given Hilbert space $H$ denote by $\Gamma(H)=\mathbb{C} \oplus H$ the boolean Fock space over $H$. The vector $\Omega=\left(\begin{array}{l}1 \\ 0\end{array}\right)$ is called the vacuum vector. The vacuum expectation $\Phi_{\Omega}: B(\Gamma(H)) \rightarrow \mathbb{C}$ is the state defined by

$$
\Phi_{\Omega}(A):=\langle\Omega, A \Omega\rangle_{\Gamma(H)} .
$$

Let $U \in H$, the creation and annihilation operators are bounded operators on $\Gamma(H)$ defined as

$$
A^{*}(U)\left(\begin{array}{c}
\alpha \\
V
\end{array}\right)=\left(\begin{array}{c}
0 \\
\alpha U
\end{array}\right), \quad A(U)\left(\begin{array}{c}
\alpha \\
V
\end{array}\right)=\left(\begin{array}{c}
\langle U, V\rangle_{H} \\
0
\end{array}\right)
$$


where $\alpha \in \mathbb{C}$ and $V \in H$. We have $A(U)$ and $A^{*}(U)$ are adjoint to each other on $\Gamma(H)$ and

$$
A(U) A^{*}(V)=\langle U, V\rangle_{H} P_{\Omega},
$$

where $P_{\Omega}$ is the projection on $\mathbb{C} \Omega$.

Now let $h$ be a separable Hilbert space and let $H=L^{2}\left(\mathbb{R}_{+} ; h\right)$ defined by

$$
L^{2}\left(\mathbb{R}_{+} ; h\right)=\left\{f: \mathbb{R}_{+} \rightarrow h \text { measurable } ; \int_{0}^{\infty}\|f(t)\|_{h}^{2} d t<\infty\right\} .
$$

For a given unit vector $u \in h$, let $u^{t}:=\chi_{[0, t[} \otimes u \in L^{2}\left(\mathbb{R}_{+} ; h\right)$ defined by $u^{t}(s)=$ $\chi_{[0, t]}(s) u \in h$ and call $A_{t}^{*}(u):=A^{*}\left(u^{t}\right), A_{t}(u):=A\left(u^{t}\right)$. A bounded operator $F$ on $\Gamma\left(L^{2}\left(\mathbb{R}_{+} ; h\right)\right)$ is given by

$$
F=\left(\begin{array}{cc}
\alpha & \langle\gamma| \\
|\delta\rangle & r
\end{array}\right)
$$

where

- $\alpha \in \mathbb{C}$,

- $\langle\gamma| \in B\left(L^{2}\left(\mathbb{R}_{+} ; h\right) ; \mathbb{C}\right)$ with $\gamma \in L^{2}\left(\mathbb{R}_{+} ; h\right)$ given by $\langle\gamma|(f):=\langle\gamma, f\rangle_{L^{2}\left(\mathbb{R}_{+} ; h\right)}$,

- $|\delta\rangle \in B\left(\mathbb{C} ; L^{2}\left(\mathbb{R}_{+} ; h\right)\right)$ with $\delta \in L^{2}\left(\mathbb{R}_{+} ; h\right)$ given by $\langle\delta|(\lambda):=\lambda \delta$,

- $r \in B\left(L^{2}\left(\mathbb{R}_{+} ; h\right)\right)$.

Definition 1. (1) A family of bounded operators $F=\left(F_{t}\right)_{t \geq 0}$ on $\Gamma\left(L^{2}\left(\mathbb{R}_{+} ; h\right)\right)$ is an adapted process if for any $t>0$

$$
F_{t}\left(\begin{array}{c}
\lambda \\
f
\end{array}\right)=F_{t}\left(\begin{array}{c}
\lambda \\
f \chi_{[0, t[}
\end{array}\right), \quad \text { for all }\left(\begin{array}{c}
\lambda \\
f
\end{array}\right) \in \Gamma(H)
$$

We denote by $\mathcal{L}(h)$ the subspace of $\Gamma(H)$ of all adapted processes.

(2) An element $F=\left(F_{t}\right)_{t \geq 0} \in \mathcal{L}(h)$ is said to be locally square integrable process if the map $t \mapsto \bar{F}_{t}$ from $\mathbb{R}_{+}$to $B(\Gamma(H))$ is measurable and satisfies

- $t \mapsto \alpha_{t} \in L_{\text {loc }}^{2}\left(\mathbb{R}_{+} ; \mathbb{C}\right)$,

- $t \mapsto \gamma_{t} \in L_{\text {loc }}^{2}\left(\mathbb{R}_{+} ; L^{2}\left(\mathbb{R}_{+} ; h\right)\right)$,

- $t \mapsto \delta_{t} \in L_{\text {loc }}^{2}\left(\mathbb{R}_{+} ; L^{2}\left(\mathbb{R}_{+} ; h\right)\right)$.

$W e$ denote by $\mathcal{L}^{2}(h)$ the subspace of $\mathcal{L}(h)$ of locally square integrable processes.

According to [1], for any locally square integrable process $F$, one can define stochastic integrals $\int_{0}^{t} F_{s} d A_{s}(u)$ and $\int_{0}^{t} d A_{s}^{*}(u) F_{s}$ by using Itô-Riemann sums. Furthermore, the processes $t \mapsto \int_{0}^{t} F_{s} d A_{s}(u)$ and $t \mapsto \int_{0}^{t} d A_{s}(u)^{*} F_{s}$ are adapted and continuous in norm.

2.5.2. Fock space realization of boolean unitary Brownian motion. We shall now provide a realization of the boolean unitary Brownian motion on the 
Boolean Fock space. According to [1] there exists a unique family of unitary operators $\left(U_{t}\right)_{t \in \mathbb{R}_{+}}$, solution to the stochastic differential equation

$$
d U_{t}=\left(d A_{t}(u)-d A_{t}^{*}(u)-\frac{1}{2} P_{\Omega} d t\right) U_{t}, \quad U_{0}=1
$$

given by

$$
U_{t}=\left(\begin{array}{cc}
\exp \left(\frac{-t}{2}\right) & L_{t,-u, \delta} \\
L_{t, u, \gamma_{1}}^{*} & M_{t, u, \zeta}^{*}+i d_{L^{2}\left(\mathbb{R}_{+} ; h\right)}
\end{array}\right)
$$

where

- $\gamma_{1}: s \mapsto \exp (-s / 2)$,

- $\delta_{t}()=.\exp \left(-\frac{1}{2}(t-).\right)$,

- $\zeta_{t}()=.-\exp \left(-\frac{1}{2}(t-).\right) \chi_{[0, t]}($.

and

- $L_{t, u, \gamma_{1}}: L^{2}\left(\mathbb{R}_{+} ; h\right) \rightarrow \mathbb{C}, \quad f \mapsto \int_{0}^{t}<u, f(s)>\gamma_{1}(s) d s$,

- $L_{t, u, \delta}^{*}: \mathbb{C} \rightarrow L^{2}\left(\mathbb{R}_{+} ; h\right), \quad \lambda \mapsto \lambda \delta_{t}(.) \chi_{[0, t)}()$.$u ,$

- $M_{t, u, \zeta}^{*}: L^{2}\left(\mathbb{R}_{+} ; h\right) \rightarrow L^{2}\left(\mathbb{R}_{+} ; h\right), \quad M_{t, u, \zeta}^{*}(f)(s)=\left\langle\zeta_{s}, f\right\rangle_{L^{2}\left(\mathbb{R}_{+} ; h\right)} \chi_{[0, t)}(s) u$.

For $i \geq 1$, we put the following notations

$$
\zeta_{t}^{i}(.)=(-1)^{i} \frac{(t-.)^{i-1}}{(i-1) !} \exp \left(-\frac{1}{2}(t-.)\right) \chi_{[0, t)}(.)
$$

and

$$
\delta_{t}^{i}(.)=(-1)^{i-1} \frac{(t-.)^{i-1}}{(i-1) !} \exp \left(-\frac{1}{2}(t-.)\right)
$$

where we put $\zeta_{t}^{1}()=.\zeta_{t}($.$) and \delta_{t}^{1}()=.\delta_{t}($.$) .$

We need the following results.

Lemma 3. For any $i \geq 1$,

$$
\begin{aligned}
& \left(M_{t, u, \zeta}^{*}\right)^{i}=M_{t, u, \zeta^{i}}^{*}, \\
& L_{t,-u, \delta} \circ M_{t, u, \zeta^{i}}^{*}=L_{t,-u, \delta^{i+1}}, \\
& L_{t,-u, \delta^{i+1}} \circ L_{t, u, \gamma_{1}}^{*}=\frac{(-t)^{i+1}}{(i+1) !} \exp (-t / 2) .
\end{aligned}
$$


Proof Let $f \in L^{2}\left(\mathbb{R}_{+} ; h\right)$. We prove the first equality by induction on $i$. The case $i=1$ is obvious. Assume the result hold for $i$, then we have

$$
\begin{aligned}
& \left(M_{t, u, \zeta}^{*}\right)^{i+1}(f)=M_{t, u, \zeta}^{*} \circ M_{t, u, \zeta^{i}}^{*}(f) \\
& =\int_{0}^{s} \exp \left(\frac{-1}{2}(.-s)\right) \int_{0}^{s}\langle u, f(r)\rangle(-1)^{i} \frac{(s-r)^{i-1}}{(i-1) !} \exp \left(-\frac{1}{2}(s-r)\right) d r \chi_{[0, t)}(s) d s \chi_{[0, t)}(.) u \\
& =\int_{0}^{\cdot}\langle u, f(r)\rangle \exp \left(\frac{-1}{2}(.-r)\right) \int_{r}(-1)^{i} \frac{(s-r)^{i-1}}{(i-1) !} \chi_{[0, t)}(s) d s d r \chi_{[0, t)}(.) u \\
& =\int_{0}\langle u, f(r)\rangle \exp \left(\frac{-1}{2}(.-r)\right)(-1)^{i+1} \frac{(.-r)^{i}}{i !} d r \chi_{[0, t)}(.) u \\
& =M_{t, u, \zeta^{i+1}}^{*}(f) .
\end{aligned}
$$

For the second assertion we have

$$
\begin{aligned}
& L_{t,-u, \delta} \circ M_{t, u, \zeta^{i}}^{*}(f) \\
& =-\int_{0}^{t} \exp \left(\frac{-1}{2}(t-s)\right) \int_{0}^{s}\langle u, f(r)\rangle(-1)^{i} \frac{(s-r)^{i-1}}{(i-1) !} \exp \left(-\frac{1}{2}(s-r)\right) d r \chi_{[0, t)}(s) d s \\
& =-\int_{0}^{t}\langle u, f(r)\rangle \exp \left(\frac{-1}{2}(t-r)\right) \int_{r}^{t}(-1)^{i} \frac{(s-r)^{i-1}}{(i-1) !} \chi_{[0, t)}(s) d s d r \\
& =-\int_{0}^{t}\langle u, f(r)\rangle \exp \left(\frac{-1}{2}(t-r)\right)(-1)^{i} \frac{(t-r)^{i}}{i !} d r \\
& =L_{t,-u, \delta^{i+1}}(f)
\end{aligned}
$$

For the last assertion obseve that

$$
L_{t,-u, \delta^{i+1}} \circ L_{t, u, \gamma_{1}}^{*}=-\int_{0}^{t} \delta_{t}^{i+1}(s) \gamma_{1}(s) d s
$$

Then the equality follows from a simple computation.

Now we come to the basic theorem.

Theorem 3. The process $\left(U_{t}\right)_{t \in \mathbb{R}_{+}}$is a boolean unitary Brownian motion.

Proof We have to show that the process $\left(U_{t}\right)_{t \in \mathbb{R}_{+}}$has boolean independent increments and that these increments $U_{s t}:=U_{t} U_{s}^{\star}$ have a stationary distribution. We start with the question of boolean independent increments. For a given $u \in h$, let $d X_{t}(u)=-i\left(d A_{t}(u)-d A_{t}^{*}(u)\right)$ then from (2.1) we obtain

$$
U_{t}-U_{s}=i \int_{s}^{t} d X_{r}(u) U_{r}-\frac{1}{2} \int_{s}^{t} P_{\Omega} U_{r} d r
$$

so that,

$$
U_{s t}=1+i \int_{s}^{t} d X_{r}(u) U_{r} U_{s}^{\star}-\frac{1}{2} \int_{s}^{t} P_{\Omega} U_{r} U_{s}^{\star} d r .
$$


Thus the operator $U_{s t}-1$ lives on the time interval $[s, t)$. But since operators to disjoint time intervals are boolean independent (c.f. [1], Remark 2.2), we get the boolean independence property of the process $\left(U_{t}\right)_{t \in \mathbb{R}_{+}}$. It remains to show that $U_{s t}$ has a stationary distribution. ¿From the equality (2.2) we have $t \mapsto U_{s t}$ is obtained by solving the same equation as $U$ but with respect to the boolean Brownian motion $\left(X_{t}(u)-X_{s}(u)\right)_{t \geq s}$. This implies that the increments of $U$ have a stationary distribution. So it is enough to prove that the distribution of $U_{t}$ is $\nu_{t}$ for all $t \geq 0$. For $n \geq 1$, we put

$$
P_{\Omega} U_{t}^{n}=\left(\begin{array}{cc}
\Phi_{\Omega}\left(U_{t}^{n}\right) & K_{t, n} \\
0 & 0
\end{array}\right)
$$

Then

$$
\begin{aligned}
P_{\Omega} U_{t}^{n+1} & =P_{\Omega} U_{t}^{n}\left(\begin{array}{cc}
\exp \left(\frac{-t}{2}\right) & L_{t,-u, \delta} \\
L_{t, u, \gamma_{1}}^{*} & M_{t, u, \zeta}^{*}+i d
\end{array}\right) \\
& =\left(\begin{array}{cc}
\exp \left(\frac{-t}{2}\right) \Phi_{\Omega}\left(U_{t}^{n}\right)+K_{t, n} \circ L_{t, u, \gamma_{1}}^{*} & \Phi_{\Omega}\left(U_{t}^{n}\right) L_{t,-u, \delta}+K_{t, n} \circ\left(M_{t, u, \zeta}^{*}+i d\right) \\
0 & 0
\end{array}\right) .
\end{aligned}
$$

By a simple identification we obtain

$$
\left\{\begin{array}{l}
\Phi_{\Omega}\left(U_{t}^{n+1}\right)=\exp (-t / 2) \Phi_{\Omega}\left(U_{t}^{n}\right)+K_{t, n} \circ L_{t, u, \gamma_{1}}^{*} \\
K_{t, n+1}=K_{t, n} \circ\left(M_{t, u, \zeta}^{*}+i d\right)+\Phi_{\Omega}\left(U_{t}^{n}\right) L_{t,-u, \delta}
\end{array} .\right.
$$

The second equation of (2.3) gives by induction

$$
K_{t, n+1}=\sum_{k=1}^{n+1} \Phi_{\Omega}\left(U_{t}^{n+1-k}\right) L_{t,-u, \delta} \circ\left(M_{t, u, \zeta}^{*}+i d\right)^{k-1} .
$$

Which in turn leads to

$$
K_{t, n+1}=\sum_{k=1}^{n+1} \Phi_{\Omega}\left(U_{t}^{n+1-k}\right) \sum_{i=0}^{k-1}\left(\begin{array}{c}
k-1 \\
i
\end{array}\right) L_{t,-u, \delta} \circ\left(M_{t, u, \zeta}^{*}\right)^{i} .
$$

Then, by Lemma 3 we obtain

$$
K_{t, n+1}=\sum_{k=1}^{n+1} \Phi_{\Omega}\left(U_{t}^{n+1-k}\right) \sum_{i=0}^{k-1}\left(\begin{array}{c}
k-1 \\
i
\end{array}\right) L_{t,-u, \delta^{i+1}} .
$$

Now, by combining the last assertion of Lemma 3 and (2.3) we get

$$
\begin{aligned}
\Phi_{\Omega}\left(U_{t}^{n+1}\right) & =\exp (-t / 2) \Phi_{\Omega}\left(U_{t}^{n}\right)+\sum_{k=1}^{n} \Phi_{\Omega}\left(U_{t}^{n-k}\right) \sum_{i=0}^{k-1}\left(\begin{array}{c}
k-1 \\
i
\end{array}\right) L_{t,-u, \delta^{i+1}} \circ L_{t, u, \gamma_{1}}^{*} \\
& =\exp (-t / 2)\left[\Phi_{\Omega}\left(U_{t}^{n}\right)+\sum_{k=0}^{n-1} \Phi_{\Omega}\left(U_{t}^{n-1-k}\right) \sum_{i=0}^{k}\left(\begin{array}{c}
k \\
i
\end{array}\right) \frac{(-t)^{i+1}}{(i+1) !}\right] .
\end{aligned}
$$


We introduce now the generating function

$$
\eta(t, z)=\sum_{n=1}^{\infty} \Phi_{\Omega}\left(U_{t}^{n}\right) z^{n}, \quad|z|<1
$$

We obtain

$$
\eta(t, z)-z \exp (-t / 2)=\exp (-t / 2)\left[z \eta(t, z)+z^{2}(1+\eta(t, z)) \sum_{n=0}^{+\infty}\left(\sum_{i=0}^{n}\left(\begin{array}{l}
n \\
i
\end{array}\right) \frac{(-t)^{i+1}}{(i+1) !}\right) z^{n}\right]
$$

which simplifies to

$$
\eta(t, z)=\exp (-t / 2) z(1+\eta(t, z))\left[1+\sum_{n=0}^{+\infty}\left(\sum_{i=0}^{n}\left(\begin{array}{c}
n \\
i
\end{array}\right) \frac{(-t)^{i+1}}{(i+1) !}\right) z^{n+1}\right] .
$$

Now using the expansion

$$
\exp \left(\frac{t z}{z-1}\right)=1+\sum_{n=1}^{+\infty}\left(\sum_{i=0}^{n-1}\left(\begin{array}{c}
n-1 \\
i
\end{array}\right) \frac{(-t)^{i+1}}{(i+1) !}\right) z^{n}
$$

we derive

$$
\begin{aligned}
\eta(t, z) & =\exp (-t / 2) z(1+\eta(t, z)) \exp \left(\frac{t z}{z-1}\right) \\
& =z(1+\eta(t, z)) \exp \left(\frac{t(1+z)}{2(z-1)}\right)
\end{aligned}
$$

Finally, we have for any $t>0, \eta(t,)=.\psi_{\nu_{t}}$ and the distribution of $U_{t}$ is $\nu_{t}$. This ends the proof of Theorem 3 .

\section{Acknowledgment}

I would like to thank U. Franz, who has through his discussions greatly contributed to the present paper and T. Hasebe for his remarks and advices. I also would like to thank an anonymous referee for helpful comments and suggestions.

\section{REFERENCES}

[1] A. Ben Ghorbal, M. Schürmann. Quantum stochastic calculus on Boolean Fock space. Infinite Dimensional Analysis, Quantum Probability and Related Topics 07:04, 631-650, 2004.

[2] H. Bercovici. Multiplicative monotonic convolution. Illinois J. Math., 49(3):929-951 (electronic), 2005.

[3] H. Bercovici. On Boolean convolutions. In Operator theory 20, volume 6 of Theta Ser. Adv. Math., pages 7-13. Theta, Bucharest, 2006.

[4] P. Biane. Free Brownian motion, free stochastic calculus and random matrices. Fields. Inst. Commun., 12, Amer. Math. Soc. Providence, RI, 1-19, 1997.

[5] U. Franz. Unification of boolean, monotone, anti-monotone, and tensor independence and Lévy process. Math. Z., 243(4):779-816, 2003.

[6] U. Franz. Multiplicative monotone convolutions. Banach Center Publications, math.PR/0503602. 73:153-166, 2006. 
[7] U. Franz. Boolean convolution of probability measures on the unit circle. Analyse et probabilités, 8394, Sémin. Congr., 16, Soc. Math. France, Paris, 2008.

[8] R. L. Hudson, K. R. Parthasarathy. Quantum Ito's formula and stochastic evolution. Commun. Math. Phys. 93, 301-323, 1984.

[9] N. Muraki. Noncommutative Brownian motion in monotone Fock space. Comm. Math. Phys., Volume 183, Number 3, 557-570, 1997.

[10] K.R. Parthasarathy. A boson Fock space realisation of arcsine Brownian motion. The Indian Journal of Statistics, Vol. 61, Series A, Pt. 3, pp. 305-311, 1999.

[11] W. T. Ross. Lens Lectures on Aleksandrov-Clark measures. https://facultystaff.richmond.edu/ wross/PDF/Clark.pdf.

[12] R. Speicher and R. Woroudi. Boolean convolution. In D. Voiculescu, editor, Free probability theory. Papers from a workshop on random matrices and operator algebra free products, Toronto, Canada, March 1995, volume 12 of Fields Inst. Commun., pages 267-279. American Mathematical Society, Providence, RI, 1997.

Université de Tunis El-Manar, Laboratoire D'Analyse Mathmatiques et appliCATIONS, LR 11ES11.

E-mail address: tarek.hamdi@ipest.rnu.tn 